\title{
Latero-posterior directed migration of trunk to the concave side of the curve: a biomechanical principle in treating the three dimensional deformity of idiopathic scoliosis with a custom molded high profile TLSO
}

SH Jang ${ }^{*}$, JA Hutson

From 9th International Conference on Conservative Management of Spinal Deformities - SOSORT 2012 Annual Meeting

Milan, Italy. 10-12 May 2012

\section{Background}

The biomechanical principles that guide orthotic treatment for Idiopathic Scoliosis (IS) are not fully defined, except for the 'three point correction' principle.

\section{Aim}

This study aims to discover the underlying biomechanical principles, used by experienced orthotists, in treating the three dimensional deformity of IS, with a custom molded TLSO.

\section{Methods}

Semi-structured individual interview, and focus group methodology, were the primary methods of data collection. Detailed descriptions of orthotic treatment, for a specified case example, were obtained from seven experienced spinal orthotists; participants held an average of 16.7 years experience in IS treatment. Sessions were audiotaped, transcribed and data was analyzed using a systematic approach to identify themes. Triangulation of data was completed.

\section{Results}

Achieving a "balanced and aligned spine and trunk in all 3 planes" emerged as the primary biomechanical goal for all 7 participants (100\%). The orthotists identified specific techniques of the treatment process such as:

Assistive Technology Department, Gillette Children's Specialty Healthcare, St. Paul, MN, USA
- drawing an iliac-clavicle box on the PA $\mathrm{x}$-ray

- determining the location and the degree of forces and finding flexibility of the curve with a hand technique

- reducing lumbar lordosis during casting • de-rotating the trunk

- centering the upper torso at the axillas over the pelvis

- creating space on the lateral posterior area of the concave side of curve and the mid-posterior area of the spine

-carving the model to achieve desired forces

- applying abdominal pressure

- building a sternal extension and a trochanteric extension.

\section{Conclusion}

To achieve the biomechanical goal, and re-align the 3dimentional deformity of IS, orthotists apply de-rotational, anterior, and lateral translational forces on the lateral side of the convex curve, and create space on the side opposite the applied force. These factors result in a biomechanical principle called latero-posterior directed migration of the trunk to the concave side of the curve.

Published: 3 June 2013

\section{Reference}

. SE K: We hold this treasure. Afton: Afton Historical Society Press; 1998.

doi:10.1186/1748-7161-8-S1-P13

Cite this article as: Jang and Hutson: Latero-posterior directed migration of trunk to the concave side of the curve: a biomechanical principle in treating the three dimensional deformity of idiopathic scoliosis with a custom molded high profile TLSO. Scoliosis 2013 8(Suppl 1):P13. 